\title{
Photonic crystals with a degenerate band edge: Field enhancement effects and sensitivity analysis
}

\author{
K.-Y. Jung and F. L. Teixeira \\ ElectroScience Laboratory and Department of Electrical and Computer Engineering, The Ohio State University, 1320 Kinnear Road, \\ Columbus, Ohio 43212, USA
}

(Received 15 October 2007; revised manuscript received 18 January 2008; published 10 March 2008)

\begin{abstract}
Photonic crystals (PhCs) with a degenerate band edge (DBE) are dispersion-tailored materials composed of a periodic arrangement of misaligned anisotropic dielectric layers and an isotropic dielectric layer. DBE PhCs yield an (approximately) quartic dispersion relation just below the DBE frequency rather than the conventional quadratic dispersion relation present below a regular band edge (RBE). We present a sensitivity analysis of the performance of DBE PhCs under various perturbations. This analysis is made possible by means of an unconditionally stable finite-difference time-domain scheme to solve Maxwell's equations in anisotropic media based on a complex-envelope alternating-direction-implicit algorithm. Numerical simulations are performed to examine the gigantic field intensity enhancement at Fabry-Perot resonances associated with finite-stack DBE PhCs. Numerical simulations are also used to investigate the sensitivity of field enhancement effects against the bandwidth of the excitation. The effects of layer thickness perturbations, grounding, and dielectric losses on the electromagnetic response of DBE PhCs are also examined. The numerical results are used to compare FabryPerot RBE resonances against Fabry-Perot DBE resonances.
\end{abstract}

DOI: $10.1103 /$ PhysRevB.77.125108

PACS number(s): 42.70.Qs, 78.20.Bh, 41.20.Jb

\section{INTRODUCTION}

Dispersion-engineered materials have attracted much attention for a myriad of applications at microwave, mm-wave, and optical frequency ranges. Periodic media such as photonic crystals ${ }^{1-10}(\mathrm{PhCs})$ are of great interest as materials with tailored dispersion because they have simple (planar) geometries and easy frequency tunability. Magnetic photonic crystals (MPhCs) are a special class of PhCs made of misaligned anisotropic dielectric layers and ferromagnetic layers that display an asymmetric dispersion diagram $\omega(k)$ with a stationary inflection point (SIP) ${ }^{3-9}$ MPhCs have striking electrodynamic properties such as vanishing group velocity, gigantic amplitude increase (with close to unit transmittance) at the SIP frequency, and electromagnetic unidirectionality. More recently, $\mathrm{PhCs}$ with a degenerate band edge (DBE) were proposed by Figotin and Vitebskiy ${ }^{6,7,10}$ to yield a gigantic amplitude increase in nonmagnetic periodic materials. DBE PhCs can be constructed by employing, for example, two misaligned anisotropic dielectric layers and one isotropic layer in each period. DBE PhCs exhibit an approximately quartic dispersion relation just below the DBE frequency rather than the quadratic dispersion relation present below regular band edge (RBE) frequencies. In order to achieve high transmittance, Fabry-Perot resonances (associated with narrow-band transmission peaks) are exploited in finite-size periodic stacks. Finite-size DBE PhCs can produce gigantic amplitude increase at Fabry-Perot resonances with very good transmittance (matching) (Ref. 10) properties.

The detailed study of such materials-including sensitivity analysis to both geometric and material perturbations, including dielectric losses-is best done by full-wave numerical techniques, since the periodicity is broken in those cases. Among full-wave techniques for solving Maxwell's equations, the finite-difference time-domain (FDTD) method ${ }^{11-13}$ is quite suited to simulate transient electromagnetic fields in inhomogeneous anisotropic dielectrics such as DBE PhCs.
One of the main challenges in the analysis of DBE PhCs by a finite-difference technique is that the spatial discretization cell size needs to be very small in order to correctly capture the sharp field distributions inside the structure. Unfortunately, the time step size of the conventional FDTD method is limited by the Courant stability criterion, which imposes an upper bound on the time step size based on the spatial cell size. This leads to a prohibitive number of time steps for the FDTD method. An alternative choice would be to employ the alternating-direction-implicit (ADI) FDTD algorithm, ${ }^{14-17}$ which is an unconditionally stable method where the time step is not bound by the Courant criterion. However, the numerical accuracy of the ADI-FDTD algorithm deteriorates as the time step size and/or the maximum frequency increases. ${ }^{18}$ Contrary to the ADI-FDTD algorithm, the numerical accuracy of the complex-envelope (CE) ADI-FDTD algorithm ${ }^{19-22}$ is governed by the bandwidth of the excitation, not by the maximum frequency. Therefore, the CEADI-FDTD algorithm is especially suited for an analysis of DBE PhCs. Numerical aspects of CE-ADI-FDTD can be found elsewhere in Refs. 20 and 21.

In this work, we model the $\mathrm{DBE} \mathrm{PhC}$ response in detail, examining field enhancement effects and providing a sensitivity analysis under geometric perturbations and the presence of dielectric losses. The effect of a ground plane on the $\mathrm{DBE} \mathrm{PhC}$ response is also considered. The present analysis is made possible by extending the CE-ADI-FDTD algorithm to anisotropic media. The remainder of this paper is organized as follows. We first outline the basic electromagnetic properties of DBE PhCs in Sec. II. Numerical results comparing the field amplitude enhancement in $\mathrm{DBE} \mathrm{PhCs}$ and $\mathrm{RBE} \mathrm{PhCs}$, as well as the effect of a ground plane on the $\mathrm{DBE} \mathrm{PhC}$ response, are presented in Sec. III. In addition, a sensitivity analysis of the $\mathrm{DBE} \mathrm{PhC}$ response against layer thickness variations and anisotropic dielectric losses is carried out, where a further comparison against $\mathrm{RBE} \mathrm{PhC}$ is made. Concluding remarks are provided in Sec. IV. Details on the extended CE-ADI-FDTD algorithm developed here 


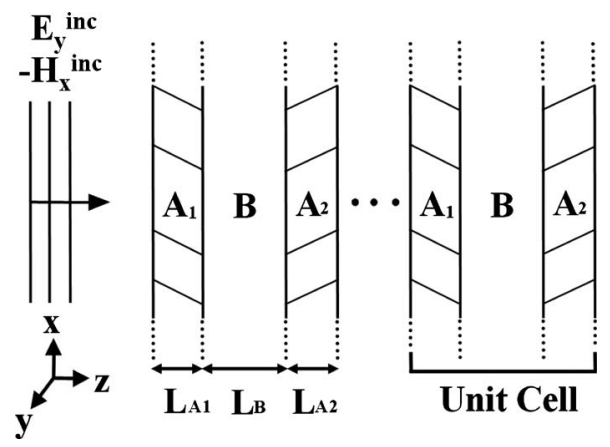

FIG. 1. Schematic of a PhC composed of two anisotropic dielectric layers ( $A$ layers) and one isotropic dielectric layer ( $B$ layer). The dispersion relation can be tailored in order to produce a degenerate band edge by adjusting the thickness and permittivity of each layer.

are included in the Appendix together with a summary of its relative computational performance.

\section{DBE PHOTONIC CRYSTALS}

We consider semi-infinite periodic media composed of two anisotropic layers ( $A$ layers) and one isotropic layer $(B$ layer) depicted in Fig. 1. Here, we assume the $B$ layer as air. With a proper choice of thickness and permittivity tensor parameters, one can tailor the dispersion relation to produce stationary points associated with either a RBE or DBE. As illustrated in the band diagrams shown in Fig. 2, the dispersion relation $\omega(k)$ can be approximated as $\omega_{b}-\omega \propto\left(k-k_{b}\right)^{4}$ just below a DBE frequency or as $\omega_{b}-\omega \propto\left(k-k_{b}\right)^{2}$ just below a RBE frequency, respectively, where $\omega_{b}$ and $k_{b}$ are the angular frequency and wave number, respectively, at the band edge. ${ }^{10}$ Below the DBE frequency, electromagnetic waves propagate at a very slow group velocity $\left[v_{g}=\partial \omega / \partial k \propto\left(\omega_{b}-\omega\right)^{3 / 4}\right]$.

In the infinite-medium case, the transmittance decreases and gradually approaches zero as the frequency $\omega$ ap-

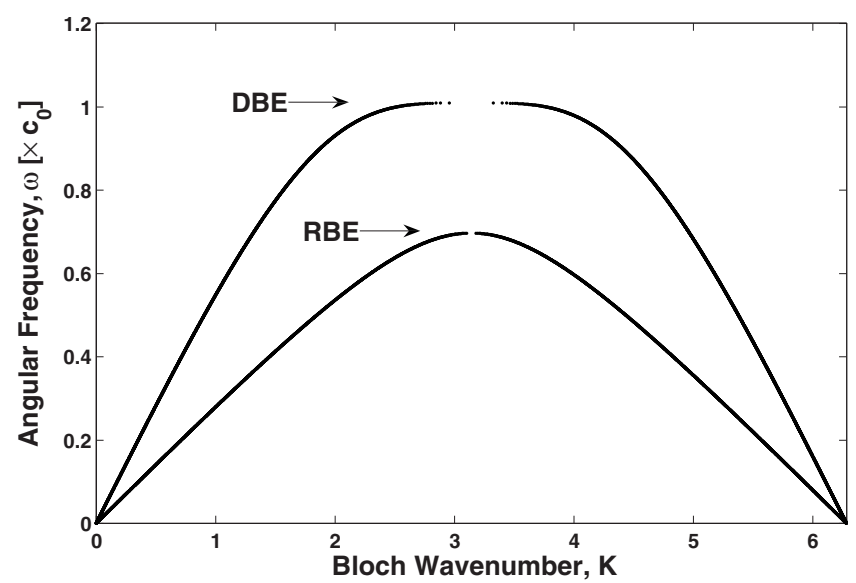

FIG. 2. Band diagram showing both a DBE and a RBE. The dispersion relation can be approximated by a quartic polynomial just below the DBE frequency and by a quadratic polynomial just below the RBE frequency (see text for details).

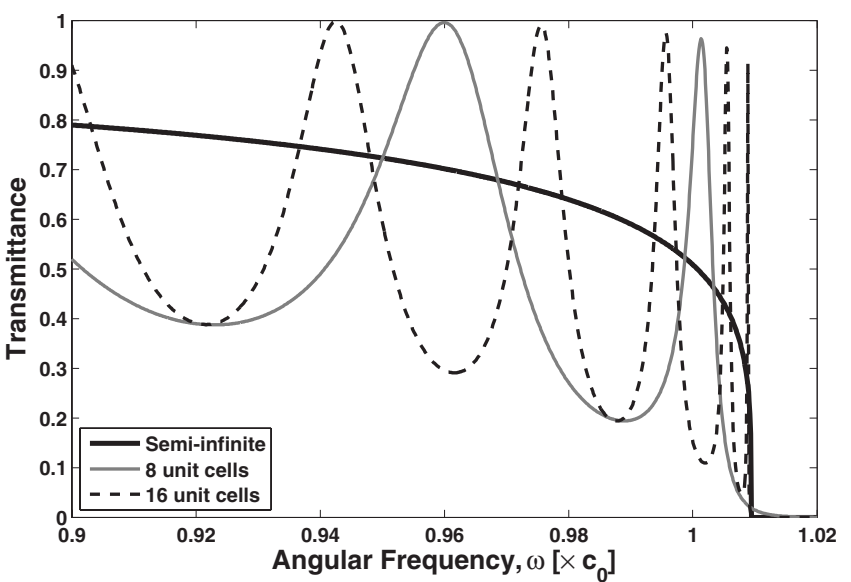

FIG. 3. Transmission spectrum of finite-stack DBE PhCs. Larger $N$ leads to Fabry-Perot resonance frequencies closer to the band edge and to narrower transmission bands.

proaches either the DBE or RBE from below. However, when a truncated (finite number of stacks) $\mathrm{DBE} \mathrm{PhC}$ is considered, Fabry-Perot cavity resonances are produced depending on the number $N$ of unit cells. The resonances below the DBE frequency are shown for $N=8$ and $N=16$ in Fig. 3. As $N$ increases, the Fabry-Perot resonance frequencies move close to the DBE frequency (while the transmission bands are narrowed). As Fabry-Perot resonances move just below the band edge, a dramatic increase in field intensity is produced because of the wave slowdown (decrease in the group velocity), similar to what occurs in MPhCs. ${ }^{4}$ Hence, a larger $N$ leads to greater growth in field intensity. We also mention here that because the group velocity is smaller below the DBE frequency (from the quartic dispersion relation) than the RBE frequency (from the quadratic dispersion relation), a much larger increase in the field amplitude is produced at DBE-based Fabry-Perot resonances than at the RBE counterpart (for same $N$ ).

\section{METHOD OF ANALYSIS}

We next examine the PhCs considered in Ref. 10 using the CE-ADI-FDTD algorithm described in the Appendix. To validate the present CE-ADI-FDTD algorithm, CE-ADIFDTD results are compared with FDTD results, showing very good agreement (see the Appendix). The accuracy of CE-ADI-FDTD results is nearly invariant unless the timestep $\Delta t$ is made close to the Nyquist limit. ${ }^{23}$ Therefore, computational time can be greatly reduced utilizing CE-ADIFDTD simulations, since one can choose a much larger $\Delta t$ for a given accuracy versus the conventional FDTD method. This makes it practical to carry out the sensitivity analysis presented in this paper.

The constitutive tensor (relative) parameters are $\epsilon_{A 1}=\epsilon_{A 2}=13.61, \quad \delta_{A 1}=\delta_{A 2}=12.4, \quad \varphi_{A 1}=0^{\circ}, \quad \varphi_{A 2}=45^{\circ}$, and $\mu_{r, A 1}=\mu_{r, A 2}=1$ [see Eq. (A10)], as considered in Ref. 10. The excitation is a raised-cosine-ramped sine wave with unit peak amplitude whose carrier angular frequency $\omega_{c}$ corresponds to the first Fabry-Perot resonance. The layer thick- 


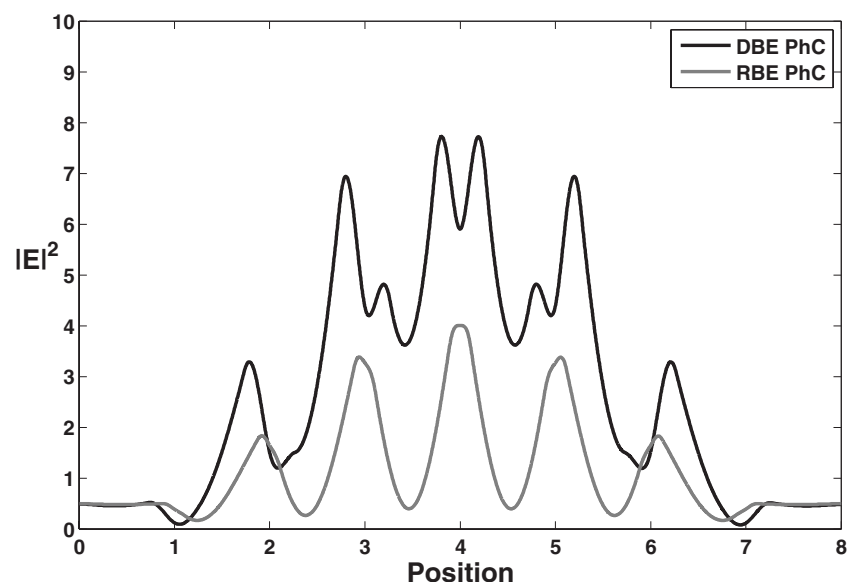

FIG. 4. Steady-state time-averaged field intensity $|\mathbf{E}|^{2}$ inside a $\mathrm{PhC}$ with $N=8$. The incident wave has unit amplitude. Further increase in the field intensity is observed in the $\mathrm{DBE} \mathrm{PhC}$ versus the $\mathrm{RBE} \mathrm{PhC}$.

nesses are as follows: $L_{A 1}=L_{A 2}=0.270545 \mathrm{~m}$ and $L_{B}$ $=0.45891 \mathrm{~m}$ for $\mathrm{DBE} \mathrm{PhCs}$ and $L_{A 1}=L_{A 2}=0.120545 \mathrm{~m}$ and $L_{B}=0.75891 \mathrm{~m}$ for RBE PhCs. This choice yields the angular frequency of the first resonance near $c_{0} \mathrm{rad} / \mathrm{s}$ for $\mathrm{DBE} \mathrm{PhCs}$ and $1.6 c_{0} \mathrm{rad} / \mathrm{s}$ for $\mathrm{RBE} \mathrm{PhCs}$, respectively, where $c_{0}$ is the numerical value of vacuum light speed in $\mathrm{m} / \mathrm{s}$. The space step is $\Delta z=1 / 400 \mathrm{~m}$, multiplied by different $\kappa$ factors inside each layer to match the respective thicknesses. We choose $\kappa_{A 1}=\kappa_{A 2}=0.270545 / 0.27$ and $\kappa_{B}$ $=0.45891 / 0.46$ for the DBE PhC simulations here. The time step size is given by $\Delta t=N_{\mathrm{C}} \kappa_{B} \Delta z / c_{0}$, where $N_{\mathrm{C}}$ is the Courant number $(\mathrm{CN})$. A Courant number $N_{\mathrm{C}}=250$ is used unless specified otherwise.

\section{INTENSITY ENHANCEMENT RESULTS}

First, we analyze a DBE $\mathrm{PhC}$ with $N=8$. Figure 4 shows the steady-state time-averaged field intensity $\left(|\mathbf{E}|^{2}\right)$ inside this finite-stack DBE PhC. The incident field has unit amplitude. Note that the field distribution has sharp variations inside the $\mathrm{DBE} \mathrm{PhC}$, which illustrates the need for a very fine mesh resolution. For comparison, we also show the results for a RBE PhC with $N=8$. The field intensity in the DBE $\mathrm{PhC}$ is about twice that of the RBE PhC.

Figure 5 shows the steady-state time-averaged $|\mathbf{E}|^{2}$ inside the DBE PhC with $N=16$ and inside the RBE PhC with $N$ $=16$. As illustrated in Fig. 3, the resonance bandwidth in this case is narrower than with $N=8$, but the resonance closer to the band edge. The gigantic enhancement in the field intensity inside the $\mathrm{DBE} \mathrm{PhC}$ is clearly visible, with the field intensity ratio of the $\mathrm{DBE} \mathrm{PhC}$ to the $\mathrm{RBE} \mathrm{PhC}$ being about 5.

We next consider the analysis of a DBE PhC with $N$ $=32$. From convergence tests, it is determined that simulations with $\Delta z=1 / 400 \mathrm{~m}$ cannot accurately analyze a DBE $\mathrm{PhC}$ that long. In this case, $\Delta z=1 / 1600 \mathrm{~m}$ is required to capture even sharper field distributions. To make the matter worse, longer time integration times should be employed to

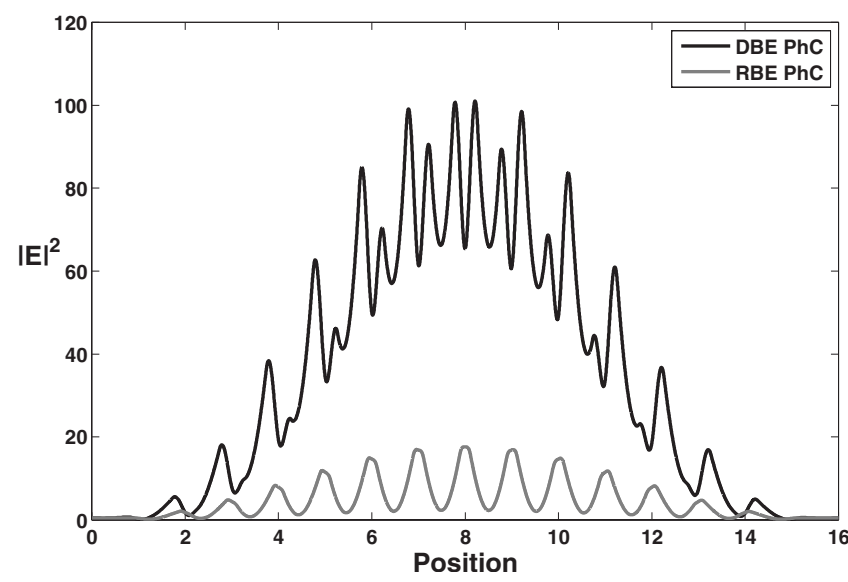

FIG. 5. Steady-state time-averaged field intensity $|\mathbf{E}|^{2}$ inside a $\mathrm{PhC}$ with $N=16$. The incident wave has unit amplitude. Further increase in the field intensity is observed in the $\mathrm{DBE} \mathrm{PhC}$ versus the RBE PhC.

obtain the necessary frequency resolution given the narrower Fabry-Perot resonance with $N=32$. Those two requirements-very small $\Delta z$ and long integration timesmake it very challenging to simulate $\mathrm{DBE} \mathrm{PhCs}$ with $N$ $=32$. We mention that it would not be practical to simulate this DBE PhC by either the conventional FDTD or ADIFDTD algorithm under our available computing resources. For analysis of the DBE PhC with $N=32$, the CE-ADI-FDTD algorithm with $N_{\mathrm{C}}=500$ is employed. Figure 6 shows the steady-state time-averaged $|\mathbf{E}|^{2}$ inside the $\mathrm{DBE} \mathrm{PhC}$ with $N=32$ and inside the $\mathrm{RBE} \mathrm{PhC}$ with $N=32$, illustrating the great difference in the intensity increase. A curve fit for these examples indicates that the peak field intensity is proportional to about $N^{3.8}$ for DBE PhCs and $N^{1.9}$ for RBE PhCs.

Next, we employ a sine-wave-modulated Gaussian pulse to illustrate the impact of the fractional bandwidth on field enhancement effects. In this case, $N_{\mathrm{C}}=50$ is employed. Fig-

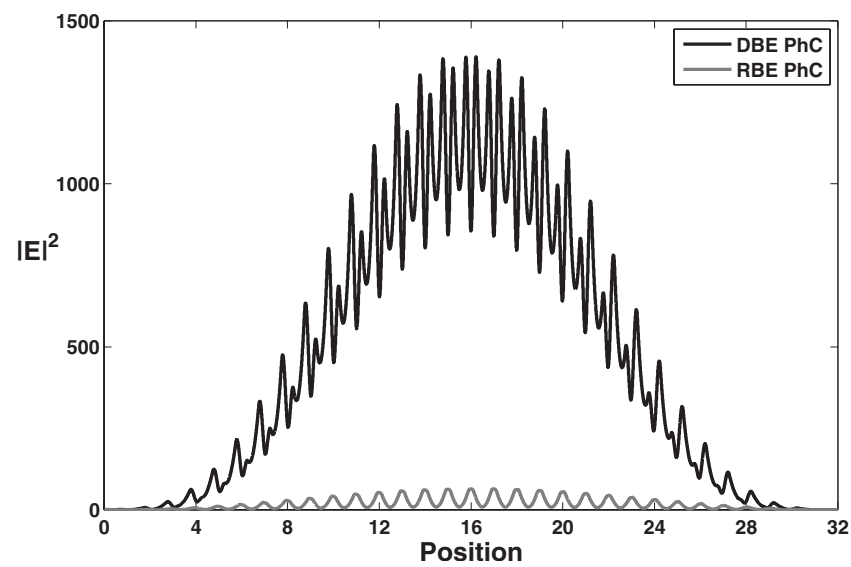

FIG. 6. Steady-state time-averaged field intensity $|\mathbf{E}|^{2}$ inside a $\mathrm{PhC}$ with $N=32$. The incident wave has unit amplitude. A much larger field intensity is observed in the $\mathrm{DBE} P \mathrm{PhC}$ versus the $\mathrm{RBE}$ $\mathrm{PhC}$. Note the larger difference between the relative intensities versus Figs. 4 and 5. 


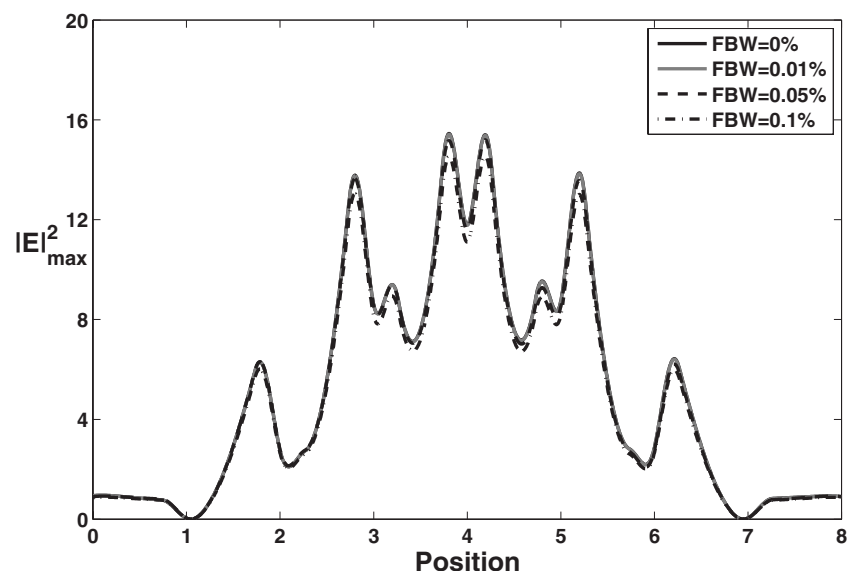

FIG. 7. Maximum field intensity $|\mathbf{E}|_{\max }^{2}$ inside the $\mathrm{DBE} \mathrm{PhC}$ with $N=8$ under sine-wave-modulated Gaussian pulse excitations. Large fractional bandwidth (FBW) leads to a decrease on field enhancement effects. Here, $\mathrm{FBW}=0 \%$ means a sine-wave excitation (without Gaussian pulse modulation).

ure 7 and Fig. 8 show the maximum field intensity $|\mathbf{E}|_{\max }^{2}$ inside the $\mathrm{DBE} \mathrm{PhC}$ with $N=8$ and inside the $\mathrm{DBE} \mathrm{PhC}$ with $N=16$, respectively. As the fractional bandwidth increases, the enhancement in the field intensity decreases. A narrower transmittance bandwidth of the Fabry-Perot resonance for $N=16$ versus $N=8$ leads to a greater impact of the fractional bandwidth on field enhancement effects (see Fig. 3).

\section{SENSITIVITY ANALYSIS}

\section{A. Ground plane effects}

In what follows, the time-domain excitation is a raisedcosine-ramped sine wave. Next, we illustrate ground plane effects on the $\mathrm{DBE} \mathrm{PhC}$ response. The ground plane is on the far end of the $\mathrm{DBE} \mathrm{PhC}$. We assume the ground plane to be a perfect electric conductor (PEC). Figure 9 shows the steady-state time-averaged $|\mathbf{E}|^{2}$ inside the PEC-backed DBE PhC with $N=8$, showing an approximately fivefold increase

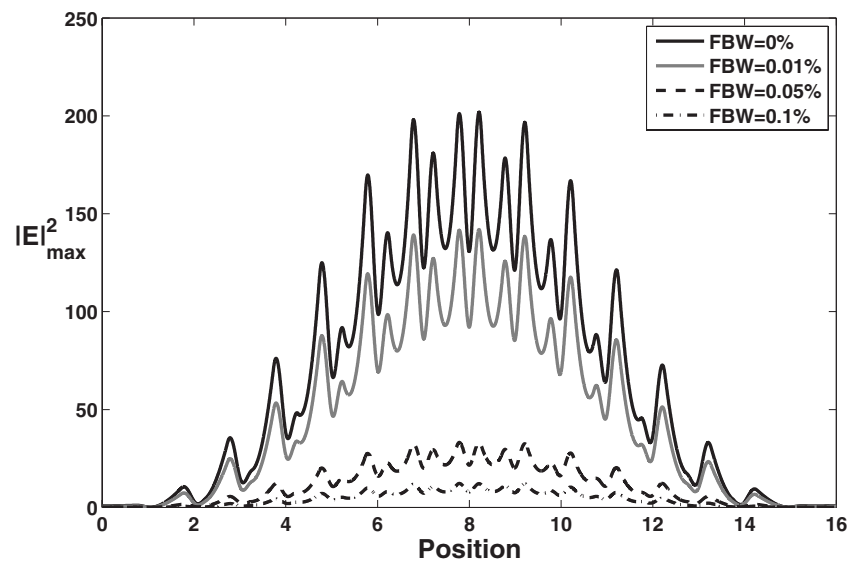

FIG. 8. Same as Fig. 7 for the DBE PhC with $N=16$. The effect of the FBW on the field intensity is more pronounced for the $N=16$ case, compared to the $N=8$ case.

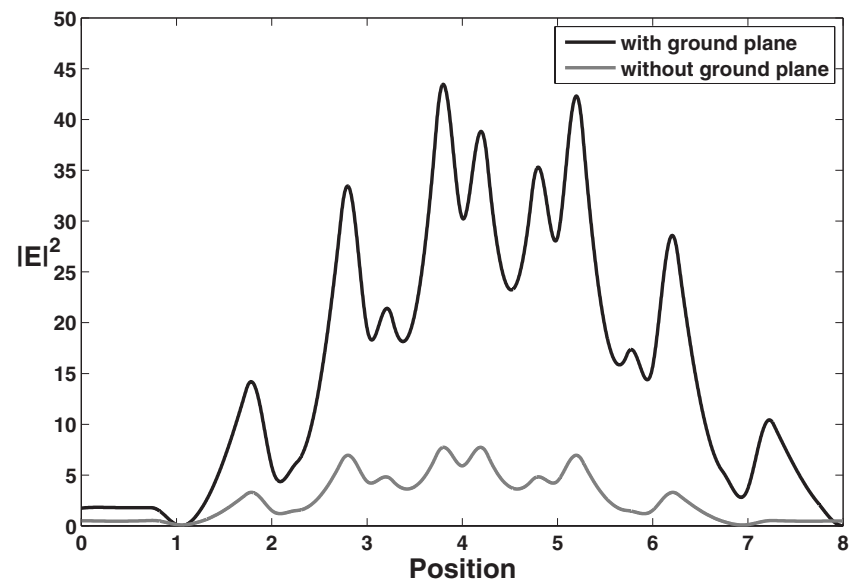

FIG. 9. Ground plane effect on the response of the DBE PhC with $N=8$. The relative increase in the field intensity due to the ground plane is about fivefold. Note that the field distribution becomes asymmetric.

in the field intensity compared to the case with no ground plane. We note that the spatial distributions in field intensity are not symmetric anymore. We also examine the effects of the ground plane on the $\mathrm{DBE} \mathrm{PhC}$ with $N=16$ and $N=32$. A similar increase of the field intensity and nonsymmetric field intensity distributions results from including a ground plane, as shown in Figs. 10 and 11. The peak intensity increase due to the ground plane is $5.63,4.03$, and 4.04 for $N=8, N=16$, and $N=32$, respectively. The increase in the field intensity can be attributed to (higher) resonance frequencies closer to the DBE frequency, thus having slower group velocity.

\section{B. Layer thickness perturbations}

Next, we assess the sensitivity of $\mathrm{PhC}$ responses to layer thickness perturbations. For this purpose, we randomly perturb the thickness of all cells for the $\mathrm{PhC}$ with $N=16$. The thickness of each cell is assumed to be a (independent)

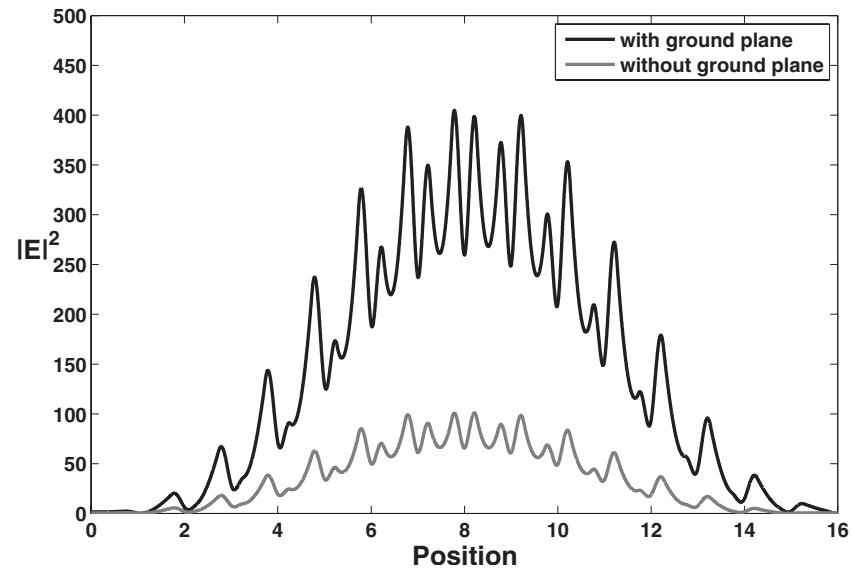

FIG. 10. Ground plane effect on the response of the DBE PhC with $N=16$. The relative increase in the field intensity due to the ground plane is about fourfold. Note that the field distribution becomes slightly asymmetric. 


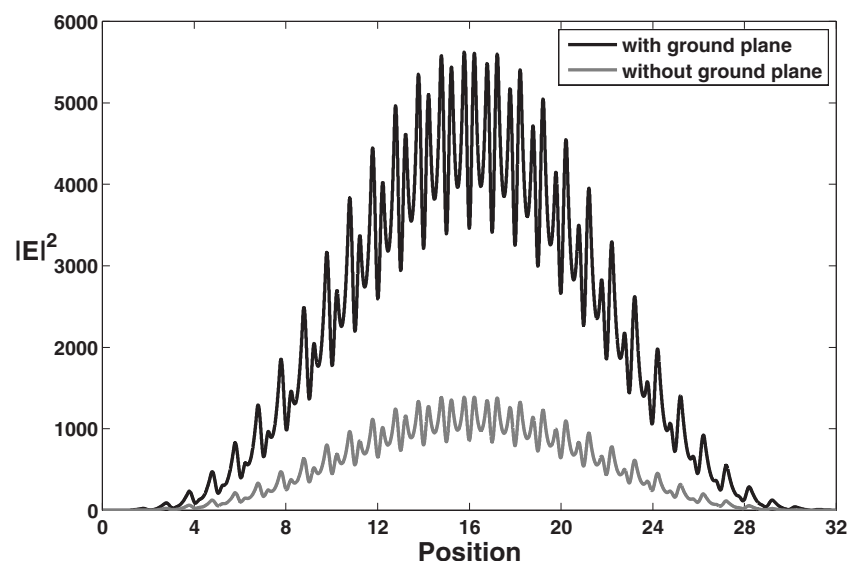

FIG. 11. Ground plane effect on the response of the DBE PhC with $N=32$. The relative increase in the field intensity due to the ground plane is again about fourfold.

Gaussian random variable centered on the nominal thickness. For each variance, an ensemble with 12 realizations is considered. Figure 12 shows the steady-state time-averaged $|\mathbf{E}|^{2}$ inside the DBE PhC with the perturbed layer thickness. As can be seen from this figure, small variations in the layer thickness can have a strong impact on the $\mathrm{DBE} \mathrm{PhC}$ performance (due to the sensitivity of the Fabry-Perot resonance to geometrical parameters). Figure 13 shows the steady-state time-averaged $|\mathbf{E}|^{2}$ inside the $\mathrm{RBE} \mathrm{PhC}$ with the same thickness perturbations. It is observed that the $\mathrm{RBE} \mathrm{PhC}$ is less sensitive to the layer thickness perturbations compared to the DBE PhC. Figure 14 displays the peak value of $|\mathbf{E}|^{2}$ inside the $\mathrm{DBE} \mathrm{PhC}$ and $\mathrm{RBE} \mathrm{PhC}$ under various layer thickness perturbations and $\mathrm{PhC}$ sizes. The $\mathrm{DBE} \mathrm{PhC}$ with $N=16$ produces less enhancement in the field intensity than the RBE PhC with $N=16$ when the layer thickness is perturbed by about $0.3 \%$. We also display the peak value of $|\mathbf{E}|^{2}$ inside the RBE $\mathrm{PhC}$ with $N=38$ (which has a similar performance in terms of intensity increase to the $\mathrm{DBE} \mathrm{PhC}$ with $N=16$ ). As shown in Fig. 14, the DBE $\mathrm{PhC}$ with $N=16$ is similarly sensitive to the considered layer thickness perturbations as

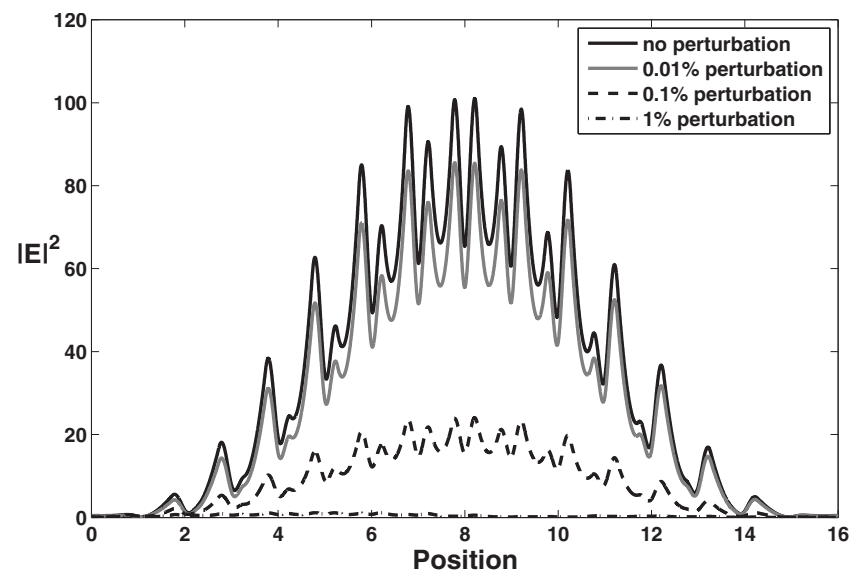

FIG. 12. Effect of layer thickness perturbations on the response of the DBE PhC with $N=16$. Small thickness perturbations lead to a decrease on field enhancement effects, as expected.

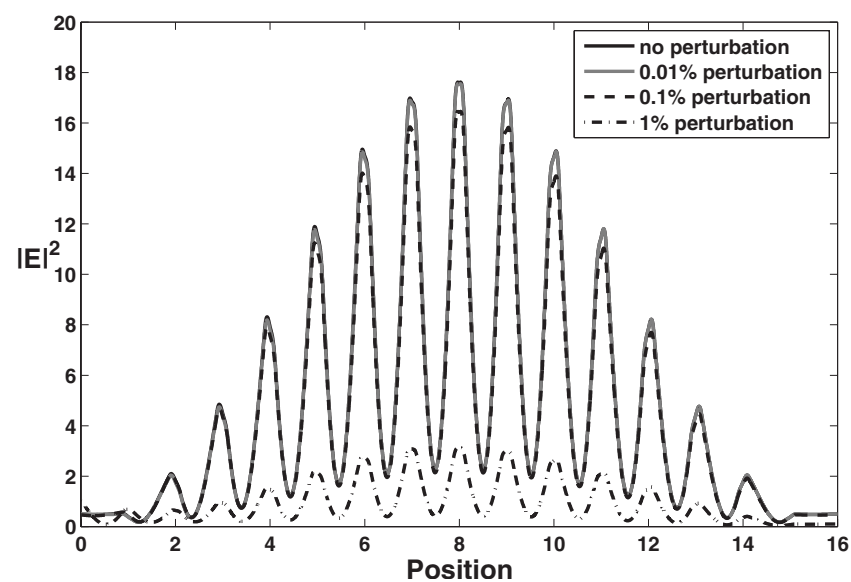

FIG. 13. Effect of layer thickness perturbations on the response of the RBE PhC with $N=16$. The decrease is relatively less pronounced than for the DBE PhC with $N=16$, but the absolute intensity enhancements are still well below those produced by the DBE $\mathrm{PhC}$ under $0.1 \%$ perturbations; see Fig. 12.

the $\mathrm{RBE} \mathrm{PhC}$ with $N=38$, with the former yielding a little bit smaller field intensity. Table I summarizes the peak value of field intensity under considered layer thickness perturbations.

\section{Dielectric losses}

The sensitivity of the $\mathrm{PhC}$ response to dielectric losses in the $A$ layers is illustrated in Fig. 15, where a loss tangent of $10^{-4}$ produces a $60 \%$ decrease in the peak field intensity in the $\mathrm{DBE} \mathrm{PhC}$ and a $10 \%$ decrease in the peak field intensity in the $\mathrm{RBE} \mathrm{PhC}$ for the same number of $N=16$ layers. Note how the $\mathrm{DBE} \mathrm{PhC}$ with $N=16$ shows a very similar sensitivity curve as the RBE PhC with $N=38$. It should be further noted that dielectric losses change the resonance peak frequency somewhat, as shown in Fig. 16. Therefore, the results presented in Fig. 15 also include any detuning effect in the

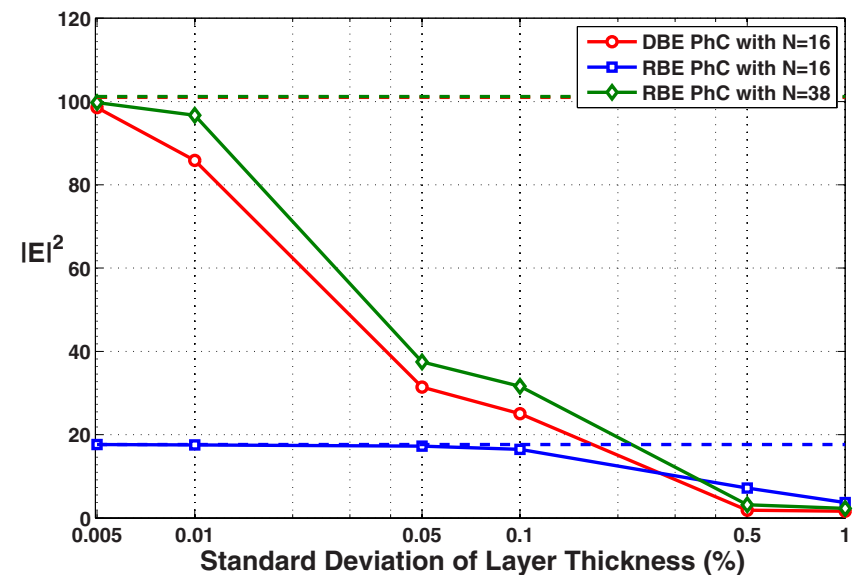

FIG. 14. (Color online) Peak value of $|\mathbf{E}|^{2}$ inside $\mathrm{PhCs}$ with layer thickness perturbations. The thickness of each cell is randomly perturbed. For each standard deviation, 12 realizations are produced and the average results for each standard deviation are plotted. The dashed lines indicate nonperturbed $\mathrm{PhC}$ results. 
TABLE I. Field intensity under layer thickness perturbations.

\begin{tabular}{lccc}
\hline \hline Perturbations & DBE $(N=16)$ & RBE $(N=16)$ & RBE $(N=38)$ \\
\hline $0.005 \%$ & 98.5167 & 17.6172 & 99.7660 \\
$0.01 \%$ & 85.8719 & 17.5628 & 96.7120 \\
$0.05 \%$ & 31.4252 & 17.2379 & 37.4918 \\
$0.1 \%$ & 25.0403 & 16.4865 & 31.6033 \\
$0.5 \%$ & 1.8743 & 7.1981 & 3.1802 \\
$1 \%$ & 1.6433 & 3.7178 & 2.3190 \\
\hline
\end{tabular}

Fabry-Perot resonances produced by the dielectric losses, since the frequency of the excitation signal is kept constant.

\section{SUMMARY AND CONCLUSION}

We examined Fabry-Perot resonances in finite-stack DBE $\mathrm{PhCs}$. The gigantic amplitude increase produced by such resonances was contrasted to similar effects yielded by RBE $\mathrm{PhCs}$. The numerical analysis has shown that the field intensity inside DBE PhCs increases approximately as $N^{3.8}$, where $N$ is the number of unit cells. It has been observed that a ground plane can boost the field intensity inside DBE PhCs. In particular, the peak field intensity inside $\mathrm{DBE}$ PhCs with $N=8$ and $N=16$, and backed by ground planes, is about 5.6 and 4 times larger, respectively, than the peak field intensity inside DBE PhCs with equal lengths.

We have performed a sensitivity analysis of $\mathrm{DBE} \mathrm{PhC}$ responses under perturbations on geometrical (layer thickness) and material (dielectric losses) parameters and compared the impact of these perturbations on the DBE and RBE $\mathrm{PhC}$ responses. As expected, the field intensity enhancement in $\mathrm{DBE}$ PhCs deteriorates under small perturbations of the layer thickness. On the other hand, the overall response is quite robust to the presence of dielectric losses. For the DBE

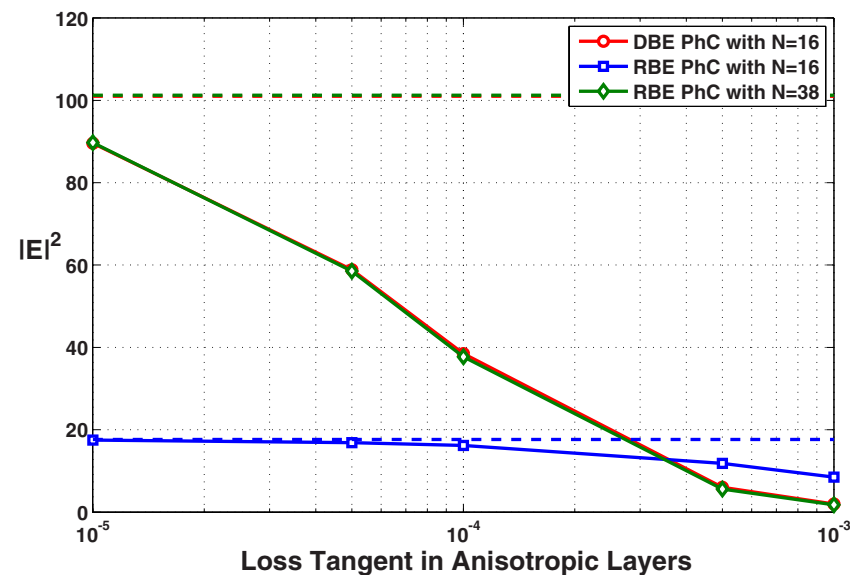

FIG. 15. (Color online) Peak value of $|\mathbf{E}|^{2}$ inside PhCs with losses in $A$ layers. The dashed lines indicate lossless $\mathrm{PhC}$ results. For the same $N$, the DBE PhC is more sensitive to dielectric losses than the RBE PhC. However, considering similar (field enhancement) performance, the DBE $\mathrm{PhC}$ with $N=16$ shows similar sensitivity to the considered dielectric losses as the RBE PhC with $N$ $=38$.

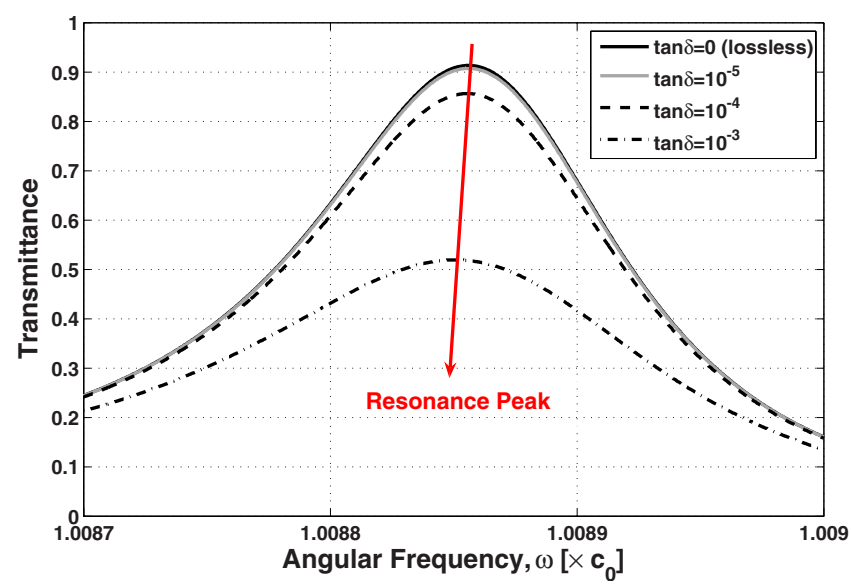

FIG. 16. (Color online) Effect of dielectric losses on the first Fabry-Perot resonance of the $\mathrm{DBE} \mathrm{PhC}$ with $N=16$. The resonance peak frequency is slightly shifted to lower frequencies by the dielectric losses.

PhC with $N=16$, a loss tangent of $10^{-5}$ decrease the peak intensity by only about $10 \%$.

A full-wave CE-ADI-FDTD algorithm has been developed to anisotropic media based on a D-H-E formulation to decouple the update of the constitutive equations from the update of the Maxwell's curl equations written in terms of (complex) field envelopes. The CE-ADI-FDTD algorithm is advantageous over both conventional FDTD and ADI-FDTD algorithms for $\mathrm{DBE} \mathrm{PhC}$ analysis, with a dramatic reduction on the overall computation time being achieved. Contrary to the traditional ADI-FDTD algorithm, the numerical dispersion error of the CE-ADI-FDTD algorithm for narrow-band problems remains small unless the time step size approaches the Nyquist limit.

\section{ACKNOWLEDGMENTS}

This work has been partially supported by the USAFOSR under MURI Grant No. FA 9550-04-1-0359, NSF under Grant No. ECS-0347502, and OSC under Grants Nos. PAS0061 and PAS-0110. K.-Y.J. would like to thank Dr. Saehoon $\mathrm{Ju}$ for helpful discussions.

\section{APPENDIX: CE-ADI-FDTD ALGORITHM FOR ANISOTROPIC MEDIA}

In this appendix, we describe the CE-ADI-FDTD algorithm used in the analysis.

Consider an anisotropic media with anisotropy in the $x y$ plane under a $y$-polarized plane wave propagating along $z$, as illustrated in Fig. 1. Both $x$ and $y$ components should be considered simultaneously due to the cross coupling of transverse electric and magnetic components. Maxwell's curl equations are expressed as

$$
\frac{\partial}{\partial t} D_{x}+\frac{\sigma_{z}}{\epsilon_{0}} D_{x}=-\frac{\partial}{\partial z} H_{y}
$$




$$
\begin{gathered}
\frac{\partial}{\partial t} D_{y}+\frac{\sigma_{z}}{\epsilon_{0}} D_{y}=\frac{\partial}{\partial z} H_{x}, \\
\frac{\partial}{\partial t} H_{x}+\frac{\sigma_{z}}{\epsilon_{0}} H_{x}=\frac{\partial}{\mu \partial z} E_{y}, \\
\frac{\partial}{\partial t} H_{y}+\frac{\sigma_{z}}{\epsilon_{0}} H_{y}=-\frac{\partial}{\mu \partial z} E_{x} .
\end{gathered}
$$

In the above, $\sigma_{z}$ is the artificial conductivity profile along the $z$ direction that implements a perfectly matched layer absorbing boundary condition. ${ }^{24-27}$ Conventional Maxwell's curl equations are recovered by setting $\sigma_{z}=0$.

We assume $\mathbf{F}=\operatorname{Re}\left[\tilde{\mathbf{F}} \cdot e^{j \omega_{c} t}\right]$, where $\omega_{c}$ is the carrier frequency, $\mathbf{F}$ denotes the field components, and $\widetilde{\mathbf{F}}$ denotes the corresponding complex envelope. Maxwell's curl equations in terms of the complex envelopes are expressed as

$$
\begin{gathered}
\frac{\partial}{\partial t} \widetilde{D_{x}}+j \omega_{c} \widetilde{D_{x}}+\frac{\sigma_{z}}{\epsilon_{0}} \widetilde{D}_{x}=-\frac{\partial}{\partial z} \widetilde{H_{y}}, \\
\frac{\partial}{\partial t} \widetilde{D_{y}}+j \omega_{c} \widetilde{D_{y}}+\frac{\sigma_{z}}{\epsilon_{0}} \widetilde{D_{y}}=\frac{\partial}{\partial z} \widetilde{H_{x}}, \\
\frac{\partial}{\partial t} \widetilde{H_{x}}+j \omega_{c} \widetilde{H}_{x}+\frac{\sigma_{z}}{\epsilon_{0}} \widetilde{H}_{x}=\frac{\partial}{\mu \partial z} \widetilde{E_{y}}, \\
\frac{\partial}{\partial t} \widetilde{H_{y}}+j \omega_{c} \widetilde{H_{y}}+\frac{\sigma_{z}}{\epsilon_{0}} \widetilde{H_{y}}=-\frac{\partial}{\mu \partial z} \widetilde{E_{x}} .
\end{gathered}
$$

The relevant constitutive equation is written as

$$
\tilde{\mathbf{D}}=[\epsilon] \tilde{\mathbf{E}},
$$

with permittivity tensor of the form

$$
\begin{aligned}
{[\epsilon] } & =\epsilon_{0}\left[\begin{array}{ccc}
\epsilon_{x x} & \epsilon_{x y} & 0 \\
\epsilon_{x y} & \epsilon_{y y} & 0 \\
0 & 0 & \epsilon_{z z}
\end{array}\right] \\
& =\epsilon_{0}\left[\begin{array}{ccc}
\epsilon+\delta \cos (2 \varphi) & \delta \sin (2 \varphi) & 0 \\
\delta \sin (2 \varphi) & \epsilon-\delta \cos (2 \varphi) & 0 \\
0 & 0 & \epsilon_{z z}
\end{array}\right],
\end{aligned}
$$

where the parameter $\delta$ is the magnitude of the in-plane anisotropy and $\varphi$ is the orientation angle of the principal axis of the permittivity tensor in the $x y$ plane.

In the ADI algorithm, ${ }^{14,15}$ the update at each time step $n$ is divided into two substeps. For simplicity, we omit the tilde in update equations that follow. For the first substep, discretized equations from Maxwell's curl, Eqs. (A5)-(A8), can be written as

$$
\begin{gathered}
D_{x_{k}}^{n+1 / 2}=c_{d 1} D_{x_{k}}^{n}-c_{d 2}\left[H_{y_{k+1 / 2}}^{n}-H_{y_{k-1 / 2}}^{n}\right], \\
D_{y_{k}}^{n+1 / 2}=c_{d 1} D_{y_{k}}^{n}+c_{d 2}\left[H_{x_{k+1 / 2}}^{n+1 / 2}-H_{x_{k-1 / 2}}^{n+1 / 2}\right], \\
H_{x_{k+1 / 2}^{n+1 / 2}}^{n+}=c_{h 1} H_{x_{k+1 / 2}}^{n}+c_{h 2}\left[E_{y_{k+1}}^{n+1 / 2}-E_{y_{k}}^{n+1 / 2}\right],
\end{gathered}
$$

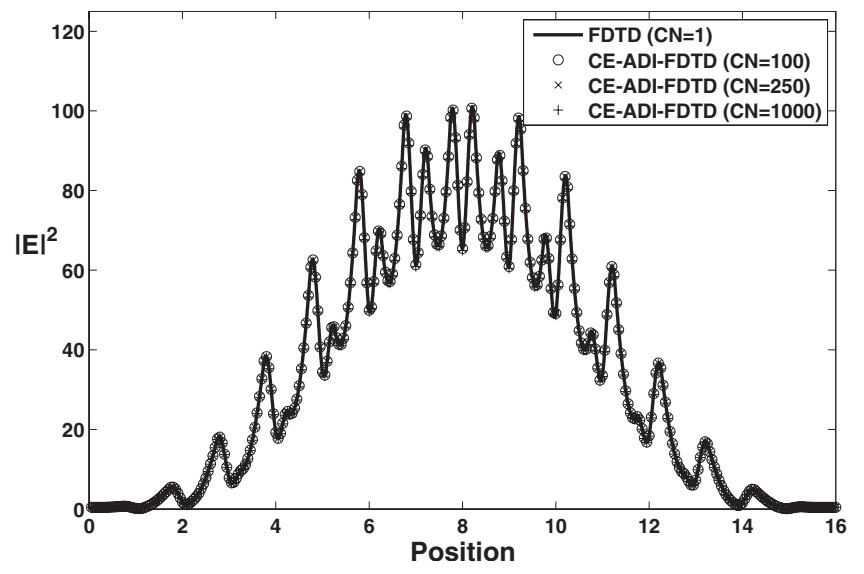

FIG. 17. CE-ADI-FDTD results using various $\mathrm{CNs}$ for the steady-state time-averaged field intensity $|\mathbf{E}|^{2}$ inside the DBE PhC with $N=16$.

$$
H_{y_{k+1 / 2}}^{n+1 / 2}=c_{h 1} H_{y_{k+1 / 2}}^{n}-c_{h 2}\left[E_{x_{k+1}}^{n}-E_{y_{k}}^{n}\right]
$$

where the subscript refers to spatial grid indexing along $z$ and the superscript refers to the time step index. The coefficients above are given by $c_{d 1}=c_{h 1}=\alpha_{-} / \alpha_{+}$and $c_{d 2}=\mu c_{h 2}=\alpha_{0} / \alpha_{+}$, where $\alpha_{ \pm}=1 \pm 0.25 \Delta t\left(j \omega_{c}+\sigma_{z} / \epsilon_{0}\right)$ and $\alpha_{0}=0.5 \Delta t / \Delta z$. The update equation for $\tilde{\mathbf{E}}$ can be obtained from the constitutive equation (A9) as

$$
\begin{aligned}
& E_{x_{k}}^{n+1 / 2}=c_{e 1 x} D_{x_{k}}^{n+1 / 2}+c_{e 2} D_{y_{k}}^{n+1 / 2}, \\
& E_{y_{k}}^{n+1 / 2}=c_{e 1 y} D_{y_{k}}^{n+1 / 2}+c_{e 2} D_{x_{k}}^{n+1 / 2},
\end{aligned}
$$

where $\quad c_{e 1 x}=\epsilon_{y y} /\left(\epsilon_{0} \Lambda_{e}\right), \quad c_{e 1 y}=\epsilon_{x x} /\left(\epsilon_{0} \Lambda_{e}\right), \quad$ and $c_{e 2}=-\epsilon_{x y} /\left(\epsilon_{0} \Lambda_{e}\right)$, with $\Lambda_{e}=\epsilon_{x x} \epsilon_{y y}-\epsilon_{x y}^{2}$. From Eq. (A12), we see that $D_{y}^{n+1 / 2}$ cannot be updated explicitly. By substituting Eq. (A16) into Eq. (A13) and plugging the resulting equation into Eq. (A12), we obtain an implicit equation for $D_{y}^{n+1 / 2}$ in a form of the tridiagonal system as follows:

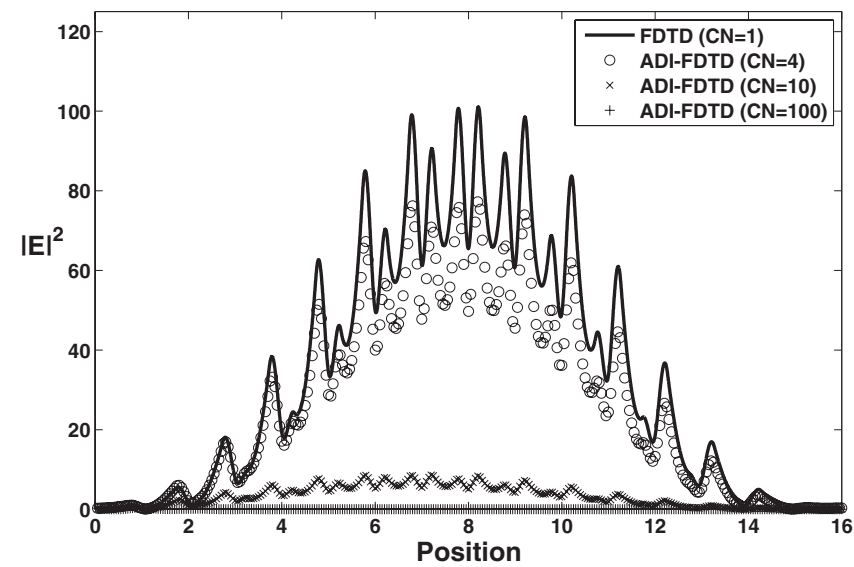

FIG. 18. ADI-FDTD results using various $\mathrm{CNs}$ for the steady-state time-averaged field intensity $|\mathbf{E}|^{2}$ inside the DBE PhC with $N=16$. 
TABLE II. Comparison between FDTD, ADI-FDTD, and CEADI-FDTD results: DBE $\mathrm{PhC}$ with $N=16$.

\begin{tabular}{lccc}
\hline \hline Method & CN & $|\mathbf{E}|_{\max }^{2}$ & CPU time \\
\hline FDTD & 1 & 201.9835 & $100 \%$ \\
ADI-FDTD & 4 & 158.0580 & $102.59 \%$ \\
ADI-FDTD & 10 & 25.6795 & $41.01 \%$ \\
ADI-FDTD & 100 & 0.73460 & $5.67 \%$ \\
CE-ADI-FDTD & 100 & 201.9235 & $13.01 \%$ \\
CE-ADI-FDTD & 250 & 201.9235 & $5.25 \%$ \\
CE-ADI-FDTD & 1000 & 200.5322 & $1.30 \%$ \\
\hline \hline
\end{tabular}

$$
\begin{aligned}
& -\gamma_{-, k} D_{y_{k-1}}^{n+1 / 2}+\left(1+\beta_{-, k}+\beta_{+_{,}}\right) D_{y_{k}}^{n+1 / 2}-\gamma_{+_{k}} D_{y_{k+1}}^{n+1 / 2} \\
& =c_{d 1_{k},{ }_{y_{k}}} D_{d 2, k}\left[c_{h 1_{k+1 / 2}} H_{x_{k+1 / 2}}^{n+1 / 2}-c_{h 1_{k-1 / 2}} H_{x_{k-1 / 2}}^{n+1 / 2}\right] \\
& +\gamma_{-, k} D_{x_{k-1}}^{n+1 / 2}-\left(\beta_{-, k}+\beta_{+_{,}}\right) D_{x_{k}}^{n+1 / 2}+\gamma_{+_{,}} D_{x_{k+1}}^{n+1 / 2} \text {, }
\end{aligned}
$$

where

$$
\begin{gathered}
\beta_{ \pm,_{k}}=c_{d 2,,_{k}} c_{h 2,,_{k \pm 1 / 2}} c_{e 1 y_{, k},}, \\
\gamma_{ \pm,_{k}}=c_{d 2,,_{k}} c_{h 2, k \pm 1 / 2} c_{e 1 y_{, k \pm 1} .} .
\end{gathered}
$$

For clarity, the spatial grid indexing is presented explicitly in the coefficients. The tridiagonal system can be efficiently solved in $O(N)$ operations. We can summarize the update procedure for the first substep as follows: (i) update $D_{x}^{n+1 / 2}$ explicitly from Eq. (A11), (ii) update $D_{y}^{n+1 / 2}$ implicitly from Eq. (A17), (iii) update explicitly $E_{x}^{n+1 / 2}$ from Eq. (A15) and $E_{y}^{n+1 / 2}$ from Eq. (A16), and (iv) update $H_{x}^{n+1 / 2}$ and $H_{y}^{n+1 / 2}$ explicitly from Eqs. (A13) and (A14), respectively.

An analogous procedure follows for the second substep. Note that the above algorithm recovers the conventional ADI-FDTD algorithm by setting the carrier frequency $\omega_{c}$ zero and by using real arithmetics. The above refers to lossless anisotropic dielectrics. The extension to lossy anisotropic dielectrics is presented in Ref. 28

Figure 17 shows the steady-state time-averaged $|\mathbf{E}|^{2}$ inside the DBE PhC with $N=16$, calculated by the CE-ADIFDTD and FDTD algorithms. Very good agreement is observed between FDTD and CE-ADI-FDTD results, for all CNs considered. On the other hand, a discrepancy is observed between ADI-FDTD and FDTD results, as shown in Fig. 18. The ADI-FDTD results start to be compromised for CNs as low as 4 . The ADI-FDTD results deteriorate as the

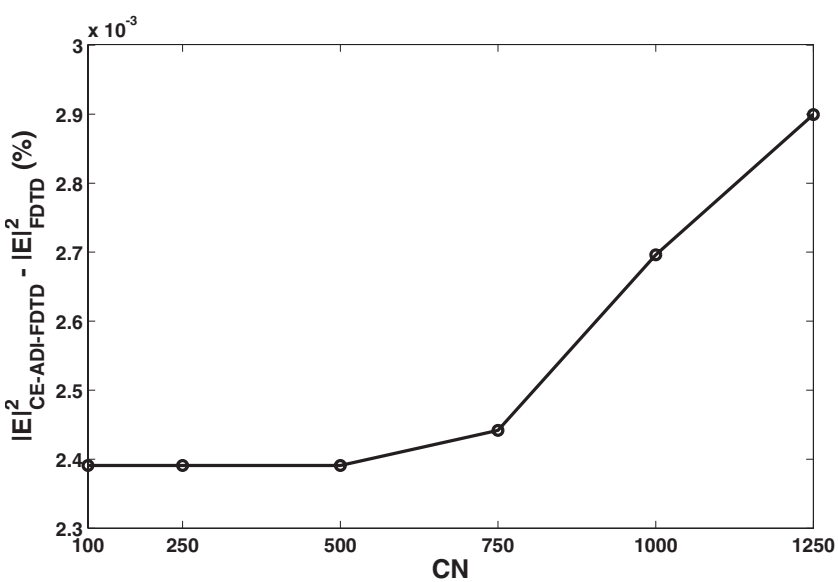

FIG. 19. Discrepancy between CE-ADI-FDTD and FDTD results for $|\mathbf{E}|^{2}$ inside the DBE PhC with $N=8$.

$\mathrm{CN}$ increases because of numerical dispersion. ${ }^{18}$

Table II summarizes the maximum value of the instantaneous field intensity $\left(|\mathbf{E}|_{\max }^{2}\right)$ inside the DBE PhC and the normalized computation time for the three methods. We notice that the maximum value of instantaneous field intensity is twice as the maximum value of time-averaged field intensity. The difference of $|\mathbf{E}|_{\max }^{2}$ between the CE-ADI-FDTD algorithm with $N_{\mathrm{C}}=250$ and the FDTD algorithm is only about $0.03 \%$. For the same $\mathrm{CN}$, the computation time of the CE-ADI-FDTD algorithm is longer than the ADI-FDTD algorithm because the former requires complex number arithmetic. Despite the increase of computation time for the same $\mathrm{CN}$, the CE-ADI-FDTD algorithm reduces computation time dramatically because it allows for much larger time steps. For example, the CPU time of the CE-ADI-FDTD algorithm with $N_{\mathrm{C}}=250$ is reduced to ca. $5.25 \%$ of the FDTD algorithm within $99.97 \%$ accuracy. In terms of memory requirements, the ADI-FDTD algorithm requires $27 \%$ more memory than the FDTD algorithm and the CE-ADI-FDTD algorithm requires $72 \%$ more memory than the FDTD algorithm, due mainly to complex arithmetic requirements.

Figure 19 shows the error in CE-ADI-FDTD results for various $\mathrm{CNs}$ in the case of the DBE PhC with $N=8$. The difference between the CE-ADI-FDTD and FDTD algorithms does not increase noticeably as the time step size is increased. In fact, the accuracy of the CE-ADI-FDTD results is nearly invariant unless $N_{\mathrm{C}} \approx 750$ (as it approaches the $\mathrm{Ny}$ quist limit). Note that CE-ADI-FDTD algorithm has very small numerical dispersion error at the carrier frequency, as pointed out in Ref. 20.
${ }^{1}$ E. Yablonovitch, Phys. Rev. Lett. 58, 2059 (1987).

${ }^{2}$ J. D. Joannopoulos, R. D. Meade, and J. N. Winn, Photonic Crystals: Molding the Flow of Light (Princeton University Press, Princeton, 1995).

${ }^{3}$ A. Figotin and I. Vitebsky, Phys. Rev. E 63, 066609 (2001).
${ }^{4}$ A. Figotin and I. Vitebskiy, Phys. Rev. B 67, 165210 (2003).

${ }^{5}$ A. Figotin and I. Vitebskiy, Phys. Rev. E 68, 036609 (2003).

${ }^{6}$ A. Figotin and I. Vitebskiy, J. Magn. Magn. Mater. 300, 117 (2006).

${ }^{7}$ A. Figotin and I. Vitebskiy, Waves Random Media 16, 293 
(2006).

${ }^{8}$ K.-Y. Jung, B. Donderici, and F. L. Teixeira, Phys. Rev. B 74, 165207 (2006).

${ }^{9}$ R. A. Chilton, K.-Y. Jung, R. Lee, and F. L. Teixeira, IEEE Trans. Microwave Theory Tech. 55, 2631 (2007).

${ }^{10}$ A. Figotin and I. Vitebskiy, Phys. Rev. E 72, 036619 (2005).

${ }^{11}$ A. Taflove and S. C. Hagness, Computational Electrodynamics: The Finite-Difference Time-Domain Method, 2nd ed. (Artech House, Norwood, MA, 2000).

${ }^{12}$ H.-T. Chien, H.-T. Tang, C.-H. Kuo, C.-C. Chen, and Z. Ye, Phys. Rev. B 70, 113101 (2004).

${ }^{13}$ A. Vial, A.-S. Grimault, D. Macias, D. Barchiesi, and M. L. de la Chapelle, Phys. Rev. B 71, 085416 (2005).

${ }^{14}$ T. Namiki, IEEE Trans. Microwave Theory Tech. 47, 2003 (1999).

${ }^{15}$ F. Zheng, Z. Chen, and J. Zhang, IEEE Microw. Guid. Wave Lett. 9, 441 (1999).

${ }^{16}$ S. Wang and F. L. Teixeira, IEEE Microw. Wirel. Compon. Lett. 13, 72 (2003).

${ }^{17}$ K.-Y. Jung and F. L. Teixeira, IEEE Photon. Technol. Lett. 19, 586 (2007).
${ }^{18}$ S. G. Garcia, T. W. Lee, and S. C. Hagness, IEEE Antennas Wireless Propag. Lett. 1, 31 (2002).

${ }^{19}$ H. Rao, R. Scarmozzino, and R. M. Osgood, Jr., IEEE Photon. Technol. Lett. 14, 477 (2002).

${ }^{20} \mathrm{~S}$. Ju, K.-Y. Jung, and H. Kim, IEEE Microw. Wirel. Compon. Lett. 13, 414 (2003).

${ }^{21}$ C. Ma and Z. Chen, IEEE Trans. Antennas Propag. 53, 971 (2005).

${ }^{22}$ O. Ramadan, IEEE Microw. Wirel. Compon. Lett. 17, 4 (2007).

${ }^{23}$ A. V. Oppenheim and R. W. Schafer, Discrete-Time Signal Processing (Prentice-Hall, Englewood Cliffs, NJ, 1989).

${ }^{24}$ J.-P. Berenger, J. Comput. Phys. 114, 185 (1994).

${ }^{25}$ W. C. Chew and W. H. Weedon, Microw. Opt. Technol. Lett. 7, 599 (1994).

${ }^{26}$ F. L. Teixeira and W. C. Chew, IEEE Microw. Guid. Wave Lett. 7, 371 (1997).

${ }^{27}$ F. L. Teixeira, K.-P. Hwang, J. Jin, and W. C. Chew, IEEE Trans. Antennas Propag. 49, 902 (2001).

${ }^{28}$ K.-Y. Jung, F. L. Teixeira, and R. Lee, IEEE Antennas Wireless Propag. Lett. 6, 643 (2007). 